\title{
How Does Antibiotherapy Affect the Clinical Outcomes of the Patients with High Prostate Specific Antigen? An Observational Study
}

\author{
(1) Oktay Özman'1, ( Zübeyr Talat ${ }^{2}$ \\ ${ }^{1}$ Gaziosmanpaşa Training and Research Hospital, Clinic of Urology, Istanbul, Turkey \\ 2 Private Clinic, Istanbul, Turkey
}

\begin{abstract}
Objective: The purpose of this observational study is to investigate the effect of urologists daily practice antibiotic usage on prostate biopsy rates and clinically insignificant cancer diagnosis rates in patients with gray-zone prostate specific antigen (PSA).

Materials and Methods: Two hundred thirty two patients who have PSA values between 2.5 and $10 \mathrm{ng} / \mathrm{mL}$ were studied prospectively. Patients convinced that he can benefit from the antibiotherapy were treated with levofloxacin 500 mg orally for 14 days by different urologist. At the end of treatment, serum PSA levels were measured again. PSA values of untreated patients were repeated after 14 days. Patients were reevaluated by same urologists with second PSA values for biopsy indication. Groups compared for biopsy rate, biopsy-to-prostate cancer detection rate, prostate cancer rate of population (Table 1, Prostate Cancer Rate 2), and Gleason 6 Prostate Cancer rate.

Results: One hundred thirty six patients were treated with levofloxacin $500 \mathrm{mg}$ for 2 weeks. Ninety six patients was untreated. PSA did not change or increased in $47(35 \%)$ of patients which treated with antibiotics. Eight-nine (65\%) patients had a decrease in PSA. Fewer biopsy indications were given in the group treated with antibiotics ( $p$ 0.001). When the all (biopsied and non-biopsied) patient groups were taken into consideration, fewer patients were diagnosed with prostate cancer in the treatment group ( $p=0.024$ ). In the treatment group Gleason 6 cancer was $29 \%$. Same rate was $45 \%$ in control group.

Conclusion: Although antibiotherapy leads urologists to make less biopsy, the rate of cancer diagnosed by biopsy does not increase.

Keywords: PSA, prostate cancer, antibiotherapy
\end{abstract}

\section{Introduction}

It is more accurate to examine the relationship between two tests performed at different time points, rather than the pointby-point evaluation of laboratory tests when making clinical decisions. Manipulation of prostate specific antigen (PSA) values, especially in the gray zone $(2.5-10 \mathrm{ng} / \mathrm{mL})$, with antibiotherapy is often the preferred clinical approach by urologists (1).

Some studies have shown that PSA may decrease with the use of antibiotics and that the biopsy requirement may be absent. The pilot study was published by Bulbul et al. (2). However, the clinical benefit of PSA reduction after antibiotherapy has not yet been clearly demonstrated. Although randomized clinical trials conducted in 2016 demonstrated a limited PSA reduction advantage with antibiotherapy, no difference was detected between positive prostate biopsy results (1).

Despite the controversial findings, antibiotherapy in the presence of high PSA continues to be frequently practiced clinically. Most clinical studies have shown that the PSA change obtained by antibiotics indicates the PSA's ability to separate prostate cancer cases from benign cases. However, there are insufficient observational studies that show which patients are specifically selected for antibiotics, or the effect of antibiotic therapy on biopsy rates and clinically insignificant cancers (Gleason 6, ISUP 1).

The purpose of this observational study was to investigate the effect of daily antibiotic use on prostate biopsy rates and clinically insignificant cancer diagnosis rates in patients with gray-zone PSA.

Cite this article as: Özman O, Talat Z. How Does Antibiotherapy Affect the Clinical Outcomes of the Patients with High Prostate Specific Antigen? An Observational Study. Bull Urooncol 2020;19(4):196-199

Address for Correspondence: Oktay Özman, Gaziosmanpaşa Training and Research Hospital, Clinic of Urology, İstanbul, Turkey Phone: +90 5545012312 E-mail: ozmanoktay@hotmail.com ORCID-ID: orcid.org/0000-0003-2499-8947 


\section{Materials and Methods}

In 2017, the clinical processes of men older than 40 years of age who applied to the urology outpatient clinics of Cerrahpaşa Faculty of Medicine were followed-up.

Ethical approval was obtained from Cerrahpaşa Faculty of Medicine Ethics Committee with the approval number: 83045809-604.01.02.

Of the 7.200 men, 3.096 (43\%) who underwent PSA screening were prospectively monitored. PSA levels of 386 patients were in the gray-zone $(2.5-10 \mathrm{ng} / \mathrm{mL})$. Fifty-four patients had a known diagnosis of prostate cancer or a recent negative pathological results detected by the transrectal ultrasound prostate biopsy$\mathrm{Bx}$ or transurethral resection of the prostate. In the follow up, 136 patients received antibiotherapy before the second PSA measurement, and the remaining 96 patients had repeated serum PSA measurements without treatment.

The patients were evaluated by different urologists. Patients convinced that they can benefit from the antibiotherapy were treated with levofloxacin $500 \mathrm{mg}$ orally for 14 days. At the end of treatment, serum PSA levels were measured again. PSA values of untreated patients were repeated after 14 days. All PSA measurements were made in the same laboratory.

Patients and their second PSA values were re-evaluated by same urologists. Twelve-quadrant systemic biopsy was performed if indicated. In addition, cognitive fusion was applied for areas where the nodule was detected by rectal examination.

\section{Statistical Analysis}

Chi-square test was used to compare the biopsy rate, biopsy-toprostate cancer detection rate, prostate cancer rate of population (Table 1, Prostate Cancer Rate ${ }_{2}$ ), and Gleason 6 prostate cancer rate in patients treated and untreated. A p-value of $<0.05$ was considered statistically significant.

\section{Results}

The study included 136 patients who were treated with levofloxacin $500 \mathrm{mg}$ for 2 weeks. Ninety-six patients who were untreated were tested again 14 days after initial evaluation. The mean ages of the patients were $65 \pm 7.9$ in the treatment group, and $62 \pm 6.4$ in the control group. There was a statistically significant difference in mean age between the two groups $(\mathrm{p}=0.007)$.

The groups were statistically similar in terms of comorbidity distribution $(p=0.36)$. The most common comorbidities were diabetes mellitus (24\%, 32/136), hypertension (31\%, 42/136), and chronic obstructive pulmonary disease $(8 \%, 11 / 136)$ in the treatment group. The same diseases were 12\% (12/96), 9\% (9/96), and 6\% (6/96), respectively, in the control group.

The use of drugs that were clinically investigated for effects on PSA, such as statins, thiazide, and aspirin, did not differ between the groups $(p=0.11)$. The groups were similar in terms of the use of other medical treatments $(p=0.09)$.

The two groups were similar in terms of lower urinary tract symptoms but not prostate volumes $(p=0.86$ and $p=0.014$, respectively). There was no statistically significant difference between groups in terms of PSA parameters (tPSA, PSA-D, and $>4 \mathrm{ng} / \mathrm{mL}$ PSA value; $\mathrm{p}=0.064, \mathrm{p}=0.6$, and $\mathrm{p}=0.68$, respectively). PSA did not change or increased in $47(35 \%)$ of patients who were treated with antibiotics. Eighty-nine (65\%) patients had a decrease in PSA. The mean PSA decrease was $1.23 \mathrm{ng} / \mathrm{mL}$. The mean PSA decline rate was $21 \%$. The mean PSA-D decrease was $0.023 \mathrm{ng} / \mathrm{mL}$. PSA decreased below the $2.5 \mathrm{ng} / \mathrm{mL}$ cut-off value in $21 \%$ of patients after treatment (Table 2).

Differences in pathologic parameters were investigated between the two groups, which were similar in terms of distribution of other factors likely to affect PSA. Accordingly, there were fewer biopsy indications in the group treated with antibiotics $(p<0.001)$. Although the rates of biopsies decreased, there was no statistically significant difference in prostate cancer detection rates among biopsied patients $(p=0.74)$. However, when both the biopsied and non-biopsied patient groups were taken into

\begin{tabular}{|c|c|c|c|}
\hline Variables & $\begin{array}{l}\text { Treatment } \\
\text { Group }\end{array}$ & $\begin{array}{l}\text { Control } \\
\text { Group }\end{array}$ & $p$-value \\
\hline Patient number & 136 & 96 & - \\
\hline Age (year, mean) & $65 \pm 7.9$ & $62 \pm 6.4$ & $0.007^{*}$ \\
\hline BMI & $27.55 \pm 2.5$ & $28.14 \pm 1.9$ & $0.052^{*}$ \\
\hline Drug usage & $46 / 136(34 \%)$ & $43 / 96(45 \%)$ & 0.09 \\
\hline NSAI & $29 / 136(21 \%)$ & $14 / 96(15 \%)$ & - \\
\hline Statin & $14 / 136(10 \%)$ & $15 / 96(16 \%)$ & 0.11 \\
\hline Tiaside & $30 / 136(22 \%)$ & $12 / 96(13 \%)$ & - \\
\hline LUTS & $88 / 136(65 \%)$ & 61/96 (64\%) & 0.86 \\
\hline Prostate volume (mL) & $58.61 \pm 22.08$ & $50.70 \pm 12.0$ & $0.014^{*}$ \\
\hline \multicolumn{4}{|l|}{ PSA Parameters } \\
\hline PSA (ng/mL, mean) & $5.38 \pm 1.56$ & $5.22 \pm 1.62$ & $0.064^{*}$ \\
\hline PSA-D (ng/mL/mL, mean) & $0.099 \pm 0.034$ & $0.104 \pm 0.025$ & $0.60^{*}$ \\
\hline$>4 \mathrm{ng} / \mathrm{mL}$ & $112 / 136(82 \%)$ & $77 / 96(80 \%)$ & 0.68 \\
\hline Biopsy rate & $47 \%(64 / 136)$ & $73 \%(70 / 96)$ & $<0.001$ \\
\hline Prostate cancer rate 1 & $38 \%(24 / 64)$ & $41 \%(29 / 70)$ & 0.74 \\
\hline Prostate cancer rate 2 & $18 \%(24 / 136)$ & $30 \%(29 / 96)$ & 0.024 \\
\hline Gleason 6 PCa rate & $29 \%(7 / 24)$ & $45 \%(13 / 29)$ & 0.24 \\
\hline \multicolumn{4}{|c|}{$\begin{array}{l}\text { *Student t-test, other } p \text { values from chi-square, statistically significance value is } \\
p<0.05 \text {, BMI: Body mass index, NSAI: Non-steroidal anti-inflammatory, LUTS: } \\
\text { Lower urinary tract symptoms, PSA: Prostate specific antigen, PCa: Prostate } \\
\text { cancer }\end{array}$} \\
\hline
\end{tabular}

\begin{tabular}{|l|l|l|}
\hline Table 2. After antibiotherapy variables & $\%$ \\
\hline Variables & \multicolumn{2}{l|}{} \\
\hline PSA Trend & $47 / 136$ & $35 \%$ \\
\hline Increase & $89 / 136$ & $65 \%$ \\
\hline Decrease & $1.23 \mathrm{ng} / \mathrm{mL}$ & - \\
\hline PSA decrease (mean) & $21 \%$ & - \\
\hline PSA decrease rate (mean) & $0.023 \mathrm{ng} / \mathrm{mL} / \mathrm{mL}$ & - \\
\hline PSA-D decrease (mean) & $19 / 89$ & $21 \%$ \\
\hline$<2.5$ ng/mL & & \\
\hline PSA: Prostate specific antigen &
\end{tabular}


consideration, fewer patients in the treatment group were diagnosed with prostate cancer $(p=0.024)$. Gleason 6 cancer rates were $29 \%$ in the treatment group and $45 \%$ in control group. Although there was less clinically insignificant cancer in the treatment group, the difference was not statistically significant $(p=0.24)$ (Table 1$)$.

\section{Discussion}

Although the contribution of PSA screening is not fully established, a clinically significant PSA reduction with antibiotherapy has been shown in many studies. In a study involving 61 patients, PSA reduction was reported in $80 \%$ of cases (3). The relatively high proportion reported in this study was attributed to the patients being diagnosed with chronic prostatitis. Patients with evidence of inflammation in prostate secretions were included in the study. This rate was $52 \%$ for the pilot study in which the effect of antibiotherapy on PSA was first investigated (2). A recently published prospective controlled trial showed that $46.5 \%$ of the patients had a PSA decrease after 3 weeks of ciprofloxacin treatment. This rate was $18 \%$ in the control group (4). In our study, $65 \%$ of the patients had PSA reduction with treatment. As shown in our study, clinically significantly PSA reduction by antibiotherapy can be obtained in approximately half of patients with a serum PSA level above $2.5 \mathrm{ng} / \mathrm{mL}$.

In our study, PSA reduction after antibiotherapy was seen to suspend clinician from biopsy indication. Prostate biopsy was performed in $47 \%$ of patients in the treatment group and $73 \%$ of patients in the control group $(p<0.001)$. There was a statistically significant difference in the final prostate cancer rates between the groups when all patients (biopsied and non-biopsied) were included $(p=0.024)$. When the groups were examined from the point of pathological results, it was seen that the rate of Gleason 6 cancer in the antibiotherapy group was less. In summary, fewer biopsies were done with antibiotics, fewer prostate cancers were diagnosed, and higher Gleason score cases were detected.

Contrary to our results, there are studies that show antibiotherapy does not have a statistically significant effect on the decision of prostate biopsy. In a study involving 108 patients older than 50 years of age with serum PSA level greater than $2.5 \mathrm{ng} / \mathrm{mL}$, prostate biopsy was performed following 3 weeks of antibiotic therapy (5). There was no significant difference between PSA parameters before and after treatment. A similar result was demonstrated in a prospective controlled study published from our country in 2013 (6). Atalay et al. (4) emphasized that even though a satisfactory decrease in PSA is obtained with antibiotherapy, it will not be enough to give up prostate biopsy. But none of these studies had an observational design. All patients underwent biopsy after antibiotherapy, regardless of the trend of PSA.

In a randomized controlled clinical trial conducted by Greiman et al. (1) $72 \%$ of the patients in the control group were biopsied and this rate was $62 \%$ in the treatment group. Similarly, in our study, antibiotherapy reduced the rate of indication for biopsy. Among the patients who underwent biopsy in the same study, $63 \%$ in the treatment group and $52 \%$ in the control group were diagnosed with prostate cancer. However, in our surveillance, there was no significant difference between the two groups
(38\% in the treatment group and $41 \%$ in the control group) with lower rates of cancer detection. That is, fewer patients were biopsied in the treatment group and prostate cancer was detected at similar rates to the control group.

A similar method was applied to work in a retrospective design published in 2009 (7). A 60\% decrease in PSA was observed in the antibiotherapy group and a biopsy of $51 \%$ was performed. The rate of detection of cancer in biopsied patients is $18 \%$. A $70 \%$ biopsy was performed in the control group and $24 \%$ of the biopsied patients had cancer. As in our study, fewer patient biopsies were performed and similar cancer rates were obtained. However, there is no data on the Gleason 6 cancer rates in this study or in the study of Greiman et al. (1).

\section{Study Limitations}

Our study has some limitations. Firstly, there was a statistically significant difference between the mean age and mean prostate volumes of the patients in the two groups. The mean age and prostate volumes of the patients in the treatment group were higher. This difference may have affected the PSA results.

\section{Conclusion}

Although antibiotherapy leads urologists to make fewer biopsies, the rate of cancer diagnosed by biopsy does not increase. Two groups with similar cancer diagnosis rates may suggest that more patients in the treatment group are away from the indication for prostate biopsy and miss cancer diagnosis. Although our study showed that Gleason 6 cancer was less common in the treatment group, it is difficult to explain this with antibiotherapy.

\section{Acknowledgements}

Publication: This study was presented in the $4^{\text {th }}$ Congress of Urologic Surgery 31 October-4 November 2020, Antalya.

Contribution: There is not any contributors who may not be listed as authors.

Conflict of Interest: No conflict of interest was declared by the authors.

Financial Disclosure: The authors declared that this study received no financial support.

\section{Ethics}

Ethics Committee Approval: For this study, approval was obtained from Cerrahpaşa University Faculty of Medicine Ethics Committee with the approval number: 83045809-604.01.02, date: 04.07.2017.

Informed Consent: Retrospective study.

Peer-review: Externally peer-reviewed.

\section{Authorship Contributions}

Concept: Z.T., O.Ö., Design: Z.T., O.Ö., Data Collection or Processing: O.Ö., Analysis or Interpretation: O.Ö., Literature Search: Z.T., O.Ö., Writing: O.Ö.

\section{References}

1. Greiman A, Shah J, Bhavsar R, et al. Six Weeks of Fluoroquinolone Antibiotic Therapy for Patients With Elevated Serum Prostate-specific 
Antigen Is Not Clinically Beneficial: A Randomized Controlled Clinical Trial. Urology 2016;90:32-37.

2. Bulbul MA, Wazzan W, Hijaz A, Shaar A. The effect of antibiotics on elevated serum prostate specific antigen in patients with urinary symptoms and negative digital rectal examination: a pilot study. J Med Liban 2002; 50:23-25.

3. Karazanashvili G, Managadze L. Prostate-specific antigen (PSA) value change after antibacterial therapy of prostate inflammation, as a diagnostic method for prostate cancer screening in cases of PSA value within $4-10 \mathrm{ng} / \mathrm{ml}$ and nonsuspicious results of digital rectal examination. Eur Urol 2001;39:538-543.

4. Atalay HA, Canat L, Alkan I, et al. Prostate-specific antigen reduction after empiric antibiotic treatment does not rule out biopsy in patients with lower urinary tract symptoms: prospective, controlled, singlecenter study. Prostate Int 2017;5:59-64.

5. Faydaci G, Eryildirim B, Tarhan F, et al. [Does antibiotherapy prevent unnecessary prostate biopsies in patients with high PSA values?]. Actas Urol Esp 2012;36:234-238.

6. Toktas G, Demiray M, Erkan E, et al. The Effect of Antibiotherapy on Prostate-Specific Antigen Levels and Prostate Biopsy Results in Patients with Levels 2.5 to $10 \mathrm{ng} / \mathrm{mL}$. J Endourol 2013;27:1061-1067.

7. Shtricker A, Shefi S, Ringel A, et al. PSA levels of $4.0-10 \mathrm{ng} / \mathrm{mL}$ and negative digital rectal examination. Antibiotic therapy versus immediate prostate biopsy. Int Braz J Urol 2009;35:551-555; discussion 555-558. 